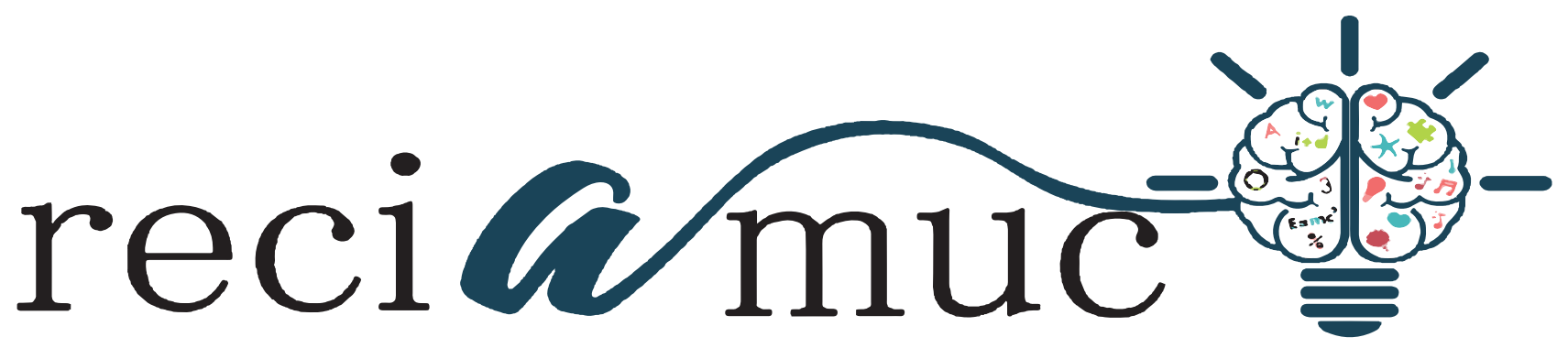

DOl: $10.26820 /$ reciamuc/4.(4).noviembre.2020.207-213

URL: https://reciamuc.com/index.php/RECIAMUC/article/view/556

EDITORIAL: Saberes del Conocimiento

REVISTA: RECIAMUC

ISSN: 2588-0748

TIPO DE INVESTIGACIÓN: Artículo de Revisión

Código UNESCO: 32 Ciencias Médicas

PAGINAS: 207-213

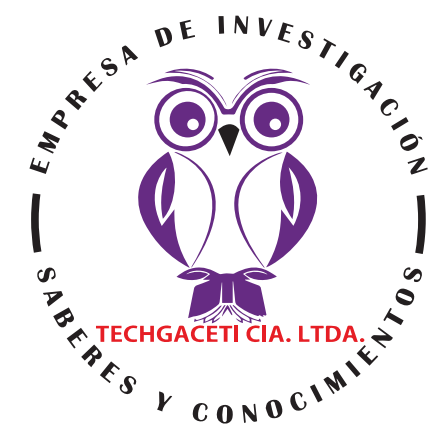

\title{
La importancia ultrasonográfica del Doppler de arteria uterina en la enfermedad hipertensiva del embarazo
}

The Ultrasonographic importance of uterine artery Doppler in hypertensive disease of pregnancy

A importância ultra-sonográfica do Doppler da artéria uterina na doença hipertensiva da gravidẻz

Silvio Eleuterio Ortiz Dueñas'; Jessenia Paola Ochoa Bustamante²; Mariana Elizabeth Hidalgo Castelo3; Arcadio Valenzuela Valencia ${ }^{4}$

RECIBIDO: 18/07/2020 ACEPTADO: 20/09/2020 PUBLICADO: 30/11/2020

1. Especialista en Ginecología y Obstetricia; Médico; Investigador Independiente; Guayaquil, Ecuador; seod801@hotmail. com; (iD) https://orcid.org/0000-0001-8080-2748

2. Master Universitario en Prevención de Riesgos Laborales; Obstetra; Médico; Investigadora Independiente; Guayaquil, Ecuador; pao_ochoab@hotmail.com; iD https://orcid.org/0000-0003-2069-0371

3. Especialista en Ginecología y Obstetricia; Médico; Investigadora Independiente; Guayaquil, Ecuador; (iD) https://orcid. org/0000-0003-3796-0390

4. Médico; Investigador Independiente; Guayaquil, Ecuador; arcavava@hotmail.com; (D) https://orcid.org/0000-0002-74007745

CORRESPONDENCIA

Silvio Eleuterio Ortiz Dueñas

seod801@hotmail.com

Guayaquil, Ecuador

๑) RECIAMUC; Editorial Saberes del Conocimiento, 2020 


\section{RESUMEN}

Las enfermedades hipertensivas del embarazo representan un grave problema de salud mundial. Estas enfermedades, en especial la preeclampsia, son las principales causas de morbilidad y mortalidad perinatal y materna. La ultrasonografía Doppler es una valoración rápidamente reproducible y confiable aún durante estadios tempranos del embarazo. El objetivo de la presente investigación consiste en plasmar, la importancia de la ecografía Doppler de arteria uterina en la enfermedad hipertensiva del embarazo, especialmente de la preeclampsia. El modelo de investigación es una revisión de tipo documental bibliográfico. La identificación de embarazadas vulnerables a desarrollar enfermedades hipertensivas, debe ser un propósito fundamental en su atención, para realizar prevención primaria. Entre los métodos de tamizaje más destacados para cumplir dicho propósito se encuentra el Doppler de arterias uterinas durante el primer y segundo trimestre. La arteria uterina es el vaso más estudiado en la evaluación Doppler en Preeclampsia, puesto que representa la condición vascular materna, a través del índice de pulsatilidad, índice de resistencia y la presencia de muesca diastólica temprana. En conclusión, este estudio ha revolucionado el concepto tradicional de la Perinatología, por cuanto ha permitido una mejor comprensión del proceso fisiopatológico tanto del del feto como de las estructuras que lo alojan. Esta comprensión resulta vital para establecer una vigilancia durante el embarazo que permita tomar las medidas necesarias para evitar complicaciones y llegar a un feliz término. En la actualidad, la ecografía Doppler de arteria uterina es una de las mejores herramientas en la predicción de enfermedades hipertensivas durante el embarazo, especialmente la preeclampsia, así como de otras complicaciones tales como: retardo en el crecimiento intrauterino (RCIU), desprendimiento placentario y muerte fetal intraútero.

Palabras clave: Importancia, Doppler, Arteria, Uterina, Embarazo.

\section{ABSTRACT}

Hypertensive diseases of pregnancy represent a serious global health problem. These diseases, especially preeclampsia, are the main causes of perinatal and maternal morbidity and mortality. Doppler ultrasonography is a rapidly reproducible and reliable assessment even during the early stages of pregnancy. The objective of this research is to show the importance of uterine artery Doppler ultrasound in hypertensive disease of pregnancy, especially pre-eclampsia. The research model is a bibliographic documentary type review. The identification of pregnant women vulnerable to developing hypertensive diseases should be a fundamental purpose in their care, to carry out primary prevention. Among the most prominent screening methods for this purpose is uterine artery Doppler during the first and second trimesters. The uterine artery is the most studied vessel in the Doppler evaluation in Preeclampsia, since it represents the maternal vascular condition, through the pulsatility index, resistance index and the presence of early diastolic notch. In conclusion, this study has revolutionized the traditional concept of Perinatology, as it has allowed a better understanding of the pathophysiological process of both the fetus and the structures that house it. This understanding is vital to establish surveillance during pregnancy that allows taking the necessary measures to avoid complications and reach a happy term. Currently, uterine artery Doppler ultrasound is one of the best tools in the prediction of hypertensive diseases during pregnancy, especially pre-eclampsia, as well as other complications such as: intrauterine growth retardation (IUGR), placental abruption and intrauterine fetal death.

Keywords: Importance, Doppler, Artery, Uterine, Pregnancy.

\section{RESUMO}

As doenças hipertensivas da gravidez representam um grave problema de saúde global. Estas doenças, especialmente a pré-eclâmpsia, são as principais causas de morbidade e mortalidade perinatal e materna. A ecografia Doppler é uma avaliação rapidamente reproduzível e fiável, mesmo durante as fases iniciais da gravidez. O objectivo desta investigação é mostrar a importância da ultra-sonografia por Doppler da artéria uterina na doença hipertensiva da gravidez, especialmente na pré-eclâmpsia. O modelo de investigação é uma revisão de tipo documental bibliográfico. A identificação de mulheres grávidas vulneráveis ao desenvolvimento de doenças hipertensivas deve ser um objectivo fundamental nos seus cuidados, para levar a cabo a prevenção primária. Entre os métodos de rastreio mais proeminentes para este fim está o Doppler da artéria uterina durante o primeiro e segundo trimestres. A artéria uterina é o vaso mais estudado na avaliação Doppler na pré-eclâmpsia, uma vez que representa a condição vascular materna, através do índice de pulsatilidade, índice de resistência e a presença de entalhes diastólicos precoces. Em conclusão, este estudo revolucionou o conceito tradicional da Perinatologia, pois permitiu uma melhor compreensão do processo fisiopatológico tanto do feto como das estruturas que o albergam. Esta compreensão é vital para estabelecer uma vigilância durante a gravidez que permita tomar as medidas necessárias para evitar complicações e alcançar um termo feliz. Actualmente, a ecografia Doppler da artéria uterina é uma das melhores ferramentas na previsão de doenças hipertensivas durante a gravidez, especialmente pré-eclâmpsia, bem como outras complicações tais como: retardamento do crescimento intra-uterino (IUGR), abrupção da placenta e morte intra-uterina do feto.

Palavras-chave: Importância, Doppler, Artéria, Uterina, Gravidez 


\section{LA IMPORTANCIA ULTRASONOGRÁFICA DEL DOPPLER DE ARTERIA UTERINA EN LA ENFERMEDAD HI- PERTENSIVA DEL EMBARAZO}

\section{Introducción}

Las enfermedades hipertensivas del embarazo representan un grave problema de salud mundial en todo el mundo. Estas enfermedades, en especial la preeclampsia, "son las principales causas de morbilidad y mortalidad perinatal y materna". (Lagunes et al., 2011, p. 213)

La hipertensión durante el embarazo puede clasificarse como:

- Crónica: la tensión arterial es alta antes del embarazo o antes de las 20 semanas de gestación. La hipertensión crónica complica el 1 a $5 \%$ de todos los embarazos.

- Gestacional: la hipertensión aparece después de las 20 semanas de gestación (típicamente, después de la semana 37) y remite después de la sexta semana posparto; aparece en el 5 o 10\% de los embarazos, más comúnmente en embarazos multifetales.

- Preeclampsia y eclampsia: Ambos tipos de hipertensión aumentan el riesgo de preeclampsia y de eclampsia, así como de otras causas de morbimortalidad materna. (Friel, 2020)

Asimismo, durante el embarazo puede desarrollarse un tipo de hipertensión llamada Hipertensión crónica con preeclampsia superpuesta. "Esta afección se presenta en las mujeres a quienes le diagnosticaron presión arterial alta crónica antes del embarazo y que luego desarrollan un empeoramiento de la presión arterial alta y proteínas en la orina, u otras complicaciones de salud durante el embarazo". (Clínica Mayo, 2018).

\section{La preeclampsia es definida como:}

La presencia de hipertensión inducida por el estado de embarazo con tensión arterial diastólica (TAD) mayor o igual a $90 \mathrm{mmHg}$ y/o tensión arterial sistólica (TAS) mayor o igual a $140 \mathrm{~mm}$ $\mathrm{Hg}$, en otros casos se definirá por un aumento de $30 \mathrm{mmHg}$ o más en la TAS o de $15 \mathrm{mmHg}$ o más en la TAD habitual, todo esto asociado con proteinuria y en ocasiones edema o lesión de órgano blanco. Según datos de la Preeclampsia Foundation, afecta el 2-10\% de los embarazos a nivel mundial siendo la principal causa de muerte materna. Puede causar parto prematuro siendo responsable por $20 \%$ de admisiones a unidades de cuidados intensivos neonatales. (Organización Panamericana de la Salud - OPS, 2019)

En un estudio realizado por la Organización Mundial de Salud y publicado en el 2014, los disturbios hipertensivos generan el 22\% aproximadamente de la mortalidad materna en América Latina, $16 \%$ en África y $12 \%$ en Asia. (Organización Mundial de Salud - OMS, 2013)

Para Lagunes et al. (2011), en la preeclampsia, los criterios diagnósticos aceptados son la proteinuria y la hipertensión arterial. No obstante, se encuentra demostrado que estos criterios suelen aparecer semanas o, incluso meses, posteriores de la invasión trofoblástica anormal y daño endotelial, que son marcadores del inicio del síndrome. En virtud de lo cual, el diagnóstico temprano de preeclampsia todavía no es posible. Frente a esta falta de recursos clínicos para diagnosticar precozmente la preeclampsia se han llevado a cabo una serie de estudios de investigación cuyo propósito es predecir su ocurrencia. (p. 214)

En tal sentido, la ecografía Doppler se ha convertido en una herramienta importante en la predicción de las enfermedades hipertensivas que se puedan presentar durante el embarazo. Se trata de un estudio imagenológico que usa ondas de sonido con el objeto de mostrar la circulación de la sangre a través de los vasos sanguíneos. Las ecografías comunes se caracterizan también por usar estas ondas de sonido para originar imágenes de estructuras internas del cuerpo, sin embargo, no son capaces de mostrar la sangre en circulación. "La ecografía Doppler funciona midiendo ondas sonoras que se reflejan en objetos en movimiento, como los glóbulos rojos. Esto se conoce como efecto Doppler". (Enciclopedia Médica ADAM, 2020)

Martínez \& Oliva (2014), acerca de la ecografía Doppler en el embarazo, refieren:

Gracias a la sofisticada tecnología ultrasonográfica, los estudios que correlacionan las mediciones del flujo y las características de la cama placentaria han proporcionado evidencia adicional de un eslabón directo de la histopatología de la arteria uterina y de la perfusión placentaria, lo cual permite la evaluación de la circulación uteroplacentaria en periodos 
tempranos de la gestación. La ultrasonografía Doppler representa una valoración rápidamente reproducible y confiable aún durante estadios tempranos del embarazo. En virtud de lo cual, es una herramienta importante en el tamizaje para el desarrollo de preeclampsia y restricción del crecimiento intrauterino para poder ser utilizado en la clínica. La detección cada vez más temprana de preeclampsia, podría representar la más alta probabilidad de conferir beneficios con una intervención Farmacológica. (p. 30)

El objetivo de la presente investigación consiste en plasmar, la importancia de la ecografía Doppler de arteria uterina en la enfermedad hipertensiva del embarazo, especialmente de la preeclampsia.

\section{Materiales y Métodos}

La presente investigación se realizó mediante una búsqueda de material bibliográfico digitalizado. Este material fue la base fundamental para el análisis y compendio del tema de la importancia ultrasonográfica del Doppler de arteria uterina en la enfermedad hipertensiva del embarazo. En razón de lo cual, la presente investigación se clasifica como de tipo documental o bibliográfica.

Se utilizaron varias bases de datos para la búsqueda entre las que destacan: SciELO, Redalyc, Medigraphic, entre otros. Asimismo, se empleó el uso de páginas web del área de la salud con reconocimiento científico mundial tales como: Organización Mundial de la Salud (OMS), Organización panamericana de la Salud (OPS), Manuales MSD, Clínica Mayo y MedlinePlus.

Para el tipo de material fueron seleccionados informes, protocolos, tesis de grado y otras clases de contenidos. Se excluyeron los contenidos repetidos, las editoriales o cartas editoriales, anotaciones académicas y todo documento carente de sustento científico o bajo nivel de evidencia.

En cuanto a los descriptores, fueron usaron los siguientes: "Doppler", "Doppler en el embarazo"; "Doppler de arteria uterina" y "Doppler de arteria uterina y enfermedades hipertensivas". La información encontrada fue filtrada bajo los criterios de idioma español, mayor relevancia y correlación temática. Igualmente, se seleccionaron aquellos trabajos de mayor actualidad (fecha de publicación de 09 años). La investiga- ción se llevó a cabo durante el mes de octubre del presente año.

\section{Resultados}

Existen factores de riesgo que predisponen a las mujeres a presentar enfermedades hipertensivas durante el embarazo. No obstante, estas enfermedades siguen siendo potencialmente imprevisibles y se carece de pruebas útiles para predecir su desarrollo en esta etapa. Urdaneta et al. (2016), explica al respecto:

...identificar mujeres vulnerables a desarrollar esta patología, debe ser un propósito fundamental ya que se requiere realizar prevención primaria en las embarazadas. Se ha descrito numerosos métodos de tamizaje, entre los que destacan los factores epidemiológicos, el Doppler de arterias uterinas durante el primer y segundo trimestre; así como diversos marcadores séricos. La arteria uterina es el vaso más estudiado en la evaluación Doppler en Preeclampsia, puesto que representa la condición vascular materna, a través del índice de pulsatilidad, índice de resistencia y la presencia de muesca diastólica temprana. (p. 4)

Asimismo, Apaza \& Delgado (2014) explican que el cambio fisiológico cardiovascular en el embarazo es el responsable del incremento del flujo sanguíneo uterino, que viene a ejercer una fuerza mayor hemodinámica sobre las paredes de los vasos y endotelio. Dicha fuerza se requiere con la finalidad de "mantener el equilibrio molecular de la relación óxido nítrico/endotelina. La consecuencia de este balance es la vasodilatación local uterina y sistémica como parte del conjunto de cambios fisiológicos cardiovasculares". Acerca de estos cambios los autores manifiestan, además:

Estos cambios se inician en el primer trimestre y se consolidan en el segundo trimestre de la gestación. Actualmente, la forma habitual de valorar el flujo sanguíneo uterino es por determinación de los índices de pulsatilidad y resistencia como expresión de la impedancia vascular, estableciendo como punto de corte el percentil 95 para una determinada edad gestacional. La tasa de detección para los índices como prueba diagnóstica tiene un amplio margen de variación, con sensibilidad que varía de 6 a 67\% y especificidad de 82 a 96\%, siendo la especificidad mejor que la sensibilidad como prueba 


\section{LA IMPORTANCIA ULTRASONOGRÁFICA DEL DOPPLER DE ARTERIA UTERINA EN LA ENFERMEDAD HI- PERTENSIVA DEL EMBARAZO}

diagnóstica. De acuerdo con diferentes estudios, el Doppler de la arteria uterina por encima de dicho punto de corte guarda relación con la presencia de RCIU, preeclampsia, desprendimiento placentario, muerte fetal intraútero. Estos resultados han sugerido mala invasión trofoblástica en el miometrio del lecho placentario y cambios fisiológicos anormales de las arterias espirales. (p. 358, 359)

Asimismo, Nodarse, Guzmán, \& Díaz (2012), explican acerca de la hipertensión arterial crónica con preeclampsia sobreañadida y la restricción de crecimiento intrauterino, que estas dos patologías son las causas principales de la morbilidad y mortalidad materna y fetal, en el mundo entero. En consecuencia, detectar estas enfermedades en una fase inicial del embarazo, es de vital importancia, por cuanto va a permitir tomar las medidas de prevención y vigilancia adecuadas y, por ende, mejorar el pronóstico, tanto para el feto como para la madre. Asimismo, explican acerca del Doppler:

El análisis de la onda de velocidad de flujo obtenida a través del flujo Doppler pulsado, es actualmente la técnica no invasiva más útil en la evaluación de las circulaciones úteroplacentarias y fetales, ya que es capaz de evidenciar un aumento de la impedancia en estas circulaciones, producto de una defectuosa invasión trofoblástica, condición compartida por la preeclampsia y el retardo del crecimiento. (p. 506)

Sáez \& Carvajal (2012), acerca del Doppler de arterias uterinas (DAUt) manifiestan lo siguiente:

Es una técnica descrita en 1983, siendo propuesta como prueba para predecir riesgo de Preeclampsia, riesgo de restricción del crecimiento fetal (RCF), y otros resultados perinatales adversos. El principio fisiopatológico de su uso se basa en la representación flujométrica en las arterias uterinas de una resistencia aumentada, dada por una invasión trofoblástica defectuosa y el fallo en la conversión de las arteriolas uterinas de alta resistencia a canales venosos de baja resistencia. Se postula que la PE de inicio precoz (antes de las 34 semanas) se basa en esta fisiopatología (invasión trofoblástica defectuosa) y se asocia más a RCF, constituyendo un cuadro severo, mientras que la preeclampsia que se manifiesta en embarazos de término no compartiría este mecanismo. La técnica puede ser por vía transvaginal (TV) o abdominal. La mayoría de los estudios de primer trimestre son por vía abdominal, aprovechando el tamizaje para aneuploidías con medición de LCN (45 a $84 \mathrm{~mm}$ ) y traslucidez de nuca que se realiza entre las 11 y 14 semanas de gestación. En el $2^{\circ}$ trimestre suele utilizarse la técnica por vía TV, aprovechando el tamizaje para parto prematuro entre las 20 y 24 semanas. Los grupos que no realizan tamizaje para parto prematuro en población general, utilizan la vía abdominal aprovechando el tamizaje de malformaciones en el mismo período del embarazo. (p. 237)

En la actualidad, como ya se ha mencionado, la forma de valorar habitualmente el flujo sanguíneo uterino es determinando los índices de pulsatilidad y resistencia como expresión de la impedancia vascular, para ello se establece como punto de corte el percentil 95 para una determinada edad gestacional. El Doppler de la arteria uterina por encima de dicho punto de corte guarda relación con:

- La presencia de retardo en el crecimiento intrauterino (RCIU)

- Preeclampsia (PE)

- Desprendimiento placentario

- Muerte fetal intraútero. (Apaza \& Delgado, 2014, p. 359)

En este orden de ideas, Apaza Valencia (2019) añade:

En general, la ecografía Doppler de la arteria uterina proporciona una predicción más precisa cuando se realiza en el segundo trimestre, que en el primer trimestre. Los índices Doppler tienen características predictivas y variables pobres, con el riesgo del paciente y la severidad de los resultados. El Doppler de arterias uterinas en el primer trimestre tiene un mejor rendimiento en la predicción de PE de inicio precoz que la PE de inicio tardío. En el segundo trimestre, en una población no seleccionada se identifica aproximadamente al 59\% de PE de inicio temprano y $60 \%$ de RCIU de inicio temprano. Como marcador aislado de enfermedad futura, la sensibilidad para predecir PE y RCIU en mujeres gestantes de riesgo bajo es moderada, de 40 a $70 \%$. (p. 166) 


\section{Conclusiones}

Del estudio se desprende que la ecografía Doppler es una gran herramienta en la predicción de complicaciones que pueden presentarse durante el embarazo y poner en riesgo la salud y la vida, tanto de la madre como del feto.

Este estudio ha revolucionado el concepto tradicional de la Perinatología, por cuanto ha permitido una mejor comprensión del proceso fisiopatológico tanto del feto como de las estructuras que lo alojan. Esta comprensión resulta vital para establecer una vigilancia durante el embarazo que permita tomar las medidas necesarias para evitar complicaciones y llegar a un feliz término.

En la actualidad, la ecografía Doppler de arteria uterina es una de las mejores herramientas en la predicción de enfermedades hipertensivas durante el embarazo, especialmente la preeclampsia, así como de otras complicaciones tales como: restricción del crecimiento intrauterino (RCIU), desprendimiento placentario y muerte fetal intraútero. Al diagnosticar doppler de arteria uterina normal en gestantes $>13$ semanas de gestación da tranquilidad a la madre por encontrarse buena circulación útero placentaria en cambio al diagnosticar doppler de arteria uterina anormal en gestantes $>13$ semanas de gestación por evidenciarse cualitativamente el notch protodiastolico y cuantitavamente por el aumento de los índice de pulsatilidad, índice de resistencia y relación sístole diástole esto se sobre entiende que no hay buena circulación útero placentaria por existir mala invasión trofoblastica. En este caso el especialista en Gineco-Obstetricia realiza el tratamiento médico por vía oral con ácido acetil salicílico 100 mg al día preferiblemente en las noches y calcio 1500 mg al día por toda la gestación para evitar la preeclampsia, restricción del crecimiento intrauterino (RCIU), desprendimiento placentario y muerte fetal intraútero.

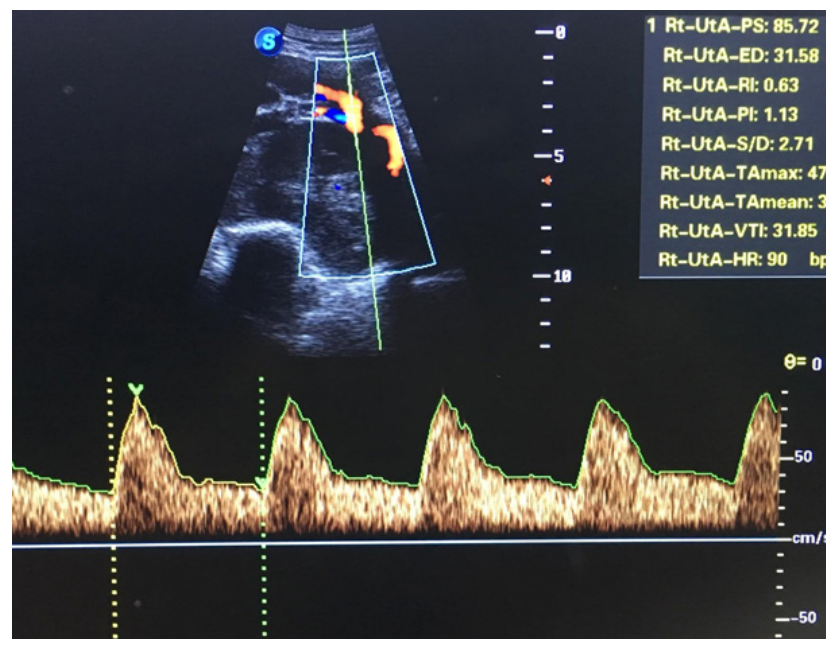

Figura 1. Doppler de arteria uterina normal en gestante $>13$ semanas de gestación

Fuente: Los autores

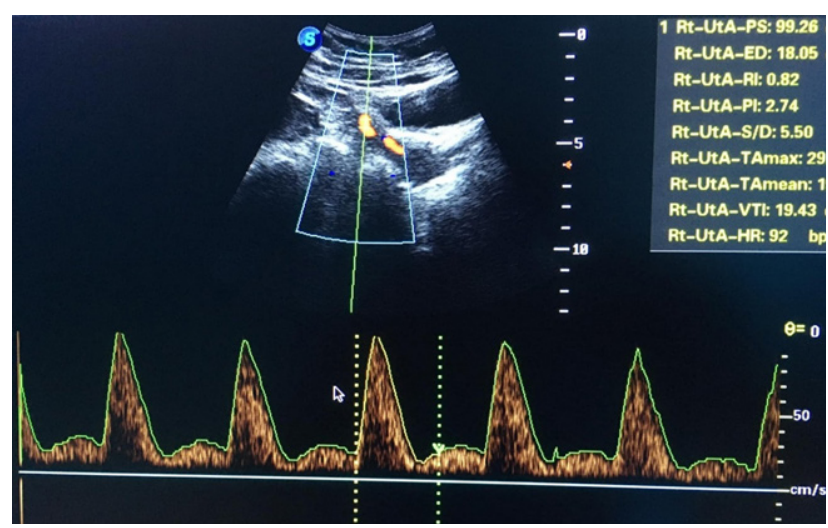

Figura 2. Doppler de arteria uterina anormal en gestante $>13$ semanas de gestación

Fuente: Los autores 


\section{LA IMPORTANCIA ULTRASONOGRÁFICA DEL DOPPLER DE ARTERIA UTERINA EN LA ENFERMEDAD HI- PERTENSIVA DEL EMBARAZO}

\section{Bibliografía}

Apaza Valencia, J. (2019). Validez diagnóstica del índice de pulsatilidad y velocidad media de la arteria uterina en preeclampsia y restricción del crecimiento intrauterino. Rev Peru Ginecol Obstet, 65(2), 163-168. doi:http:// dx.doi.org/10.31403/rpgo.v65i2166

Apaza, J., \& Delgado, J. (2014). Índices y velocidades de la arteria uterina como indicadores de hipoperfusión uterina en gestantes con restricción del crecimiento intrauterino. Revista Peruana de Ginecología y Obstetricia, 355-361. Recuperado el 22 de Octubre de 2020, de http://www.scielo.org.pe/pdf/rgo/v61n4/a02v61n4.pdf

Clínica Mayo. (16 de Noviembre de 2018). Clínica Mayo. Recuperado el 16 de Octubre de 2020, de https:// www.mayoclinic.org/es-es/diseases-conditions/preeclampsia/symptoms-causes/syc-20355745\#: :text=Hipertensi\%C3\%B3n\%20cr\%C3\%B3nica\%20con\%20 preeclampsia\%20superpuesta,de\%20salud\%20durante\%20el\%20embarazo.

Enciclopedia Médica ADAM. (31 de Julio de 2020). MedlinePlus. (B. N. UU., Editor) Recuperado el 20 de Octubre de 2020, de https://medlineplus.gov/spanish/pruebas-de-laboratorio/ecografia-doppler/

Friel, L. A. (Abril de 2020). Manuales MSD. Recuperado el 14 de Octubre de 2020, de https://www.msdmanuals. com/es-ve/professional/ginecolog\%C3\%ADa-y-obstetricia/complicaciones-no-obst\%C3\%A9tricas-durante-el-embarazo/hipertensi\%C3\%B3n-en-el-embarazo

Lagunes, A. L., Ríos, B., Peralta, M., Cruz, P., Sánchez, S., Sánchez, J., . . . López, G. (2011). Guía de práctica clínica. Enfermedades hipertensivas del embarazo. Rev Med Inst Mex Seguro Soc, 49(2), 213-224. Recuperado el 18 de Octubre de 2020, de https://www.medigraphic. com/pdfs/imss/im-2011/im112t.pdf
Martínez, P., \& Oliva, L. (2014). Flujometría Doppler en medicina materno fetal. R! evista Médica de Honduras, 82(2), 27-32. Recuperado el 20 de Octubre de 2020, de http://www.bvs.hn/RMH/pdf/2014/pdf/Vol82-1-20149.pdf

Nodarse, A., Guzmán, R., \& Díaz, D. (2012). Valor del índice de pulsatilidad promedio de las arterias uterinas en gestantes hipertensas crónicas. Revista Cubana de Obstetricia y Ginecología, 38(4), 499-509. Recuperado el 23 de Octubre de 2020, de http://scielo.sld.cu/pdf/ gin/v38n4/gin07412.pdf

Organización Mundial de Salud - OMS. (2013). Organización Mundial de Salud - OMS. Recuperado el 19 de Octubre de 2020, de https://apps.who.int/iris/bitstream/ handle/10665/119742/WHO_RHR_14.17_spa.pdf?sequence $=1$

Organización Panamericana de la Salud - OPS. (27 de Marzo de 2019). Organización Panamericana de la Salud - OPS. Recuperado el 18 de Octubre de 2020, de https://www.paho.org/clap/index.php?option=com_ content\&view $=$ article\&id=452:22-de-mayo-dia-mundial-de-la-preeclampsia\&ltemid=215\&lang=es\#: :text $=$ La $\% 20$ preeclampsia $\% 20$ se $\% 20$ define $\% 20$ como,m\%C3\%A1s\%20en\%20la\%20TAD\%20habitual\%2C

Sáez, N., \& Carvajal, J. (2012). Tamizaje y prevención de preeclampsia guiado por Doppler de arterias uterinas: revisión sistemática de la literatura. Revista Chilena de ginecología y Obstetricia, 77(3), 235-242. Recuperado el 29 de Octubre de 2020, de https://scielo.conicyt.cl/ pdf/rchog/v77n3/art11.pdf

Urdaneta, J. R., Sierralta, M., Baabel, N., Contreras, A., Valbuena, G., Labarca, L., . . Monquillaza, J. (2016). Doppler de arterias uterinas en la predicción de resultados perinatales adversos (revisión). VITAE, 68, 1-12. Recuperado el 21 de Octubre de 2020, de https://vitae. ucv.ve/pdfs/VITAE_5419.pdf

\section{CITAR ESTE ARTICULO:}

Ortiz Dueñas, S. E., Ochoa Bustamante, J. P., Hidalgo Castelo, M. E., \& Valenzuela Valencia, A. (2020). La importancia ultrasonográfica del Doppler de arteria uterina en la enfermedad hipertensiva del embarazo. RECIAMUC, 4(4).

https://doi.org/10.26820/reciamuc/4.(4).noviembre.2020.207-213

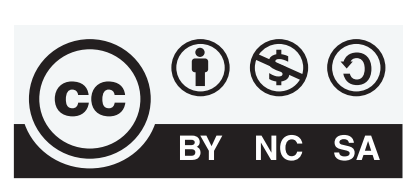

CREATIVE COMMONS RECONOCIMIENTO-NOCOMERCIAL-COMPARTIRIGUAL 4.0. 